POS $\quad$ PROCEEDINGS

\title{
Unification of Diverse AGN Outflows
}

\author{
Keigo Fukumura*t \\ James Madison University, USA \\ E-mail: fukumukxejmu.edu
}

Demos Kazanas

$N A S A / G S F C, U S A$

E-mail: demos.kazanas@nasa.gov

Francesco Tombesi

NASA/GSFC/UMD, USA

E-mail: ftombesi@astro.umd.edu

\section{Chris Shrader}

NASA/GSFC/USRA, USA

E-mail: chris.r.shraderenasa.gov

\section{Ehud Behar}

Department of Physics, Technion, Israel

E-mail: beharaphysics.technion.ac.il

\section{Ioannis Contopoulos}

Academy of Athens, Greece

E-mail: icontop@Academyofathens.gr

Diverse outflows have been detected in the proximity to many classes of active galactic nuclei (AGNs) in the past decades primarily in UV and X-ray spectroscopic observations. This presentation will focus primarily on X-ray-absorbing outflows in AGNs first showing a generic characteristics of the overall blueshifted absorbers at length scale up to pc, followed by a current status of our understanding of its physical properties and geometrical perspective. While challenging and unclear, there has been an attempt in recent years to constrain its launching mechanisms(s) and a global picture of the AGN-driven X-ray outflows. In this talk, I will review the basis of the outflow physics both in observational and theoretical standpoints and further elaborate one of the most promising candidate scenarios where plasma is magnetically launched and accelerated from a black hole accretion disk that is being photoionized by the AGN ionizing radiation field imprinting complex absorption features in X-ray spectrum. As a case study the model is applied to a bright radio-quiet quasar, PG $1211+143$, demonstrating its predictable power for various wind properties such as column density, ionization state, line-of-sight angle, AGN SED dependence and density profile of the magnetized wind.

XI Multifrequency Behaviour of High Energy Cosmic Sources Workshop -MULTIF15-,

25-30 May 2015

Palermo, Italy

\footnotetext{
*Speaker.

${ }^{\dagger}$ Also an KITP scholar at UCSB.
} 


\section{Overview of AGN Outflows: Warm Absorbers \& Ultra-Fast Outflows}

AGN-driven outflows have been intensively studied primarily in spectroscopic view for the past decades from multi-wavelength observations to constrain its physical properties and geometrical origin. Despite an increasing amount of high-quality data obtained with the state-of-the-art instruments (both CCDs and dispersive detectors), a definitive understanding of these outflows still remains vague to date. It is also critical to understand the outflows in the context of the so called AGN feedback since theories (including a number of numerical simulations) appear to imply that a certain type of AGN outflows could sufficiently deliver its kinetic power all the way to the circumnuclear regions (at pc to kpc scales) to quench star-forming activities by disrupting the cold gas which would otherwise be effectively detached from the nuclear region. Therefore, the physics of AGN outflows could potentially provide a profound insight into the AGN-galaxy co-evolution.
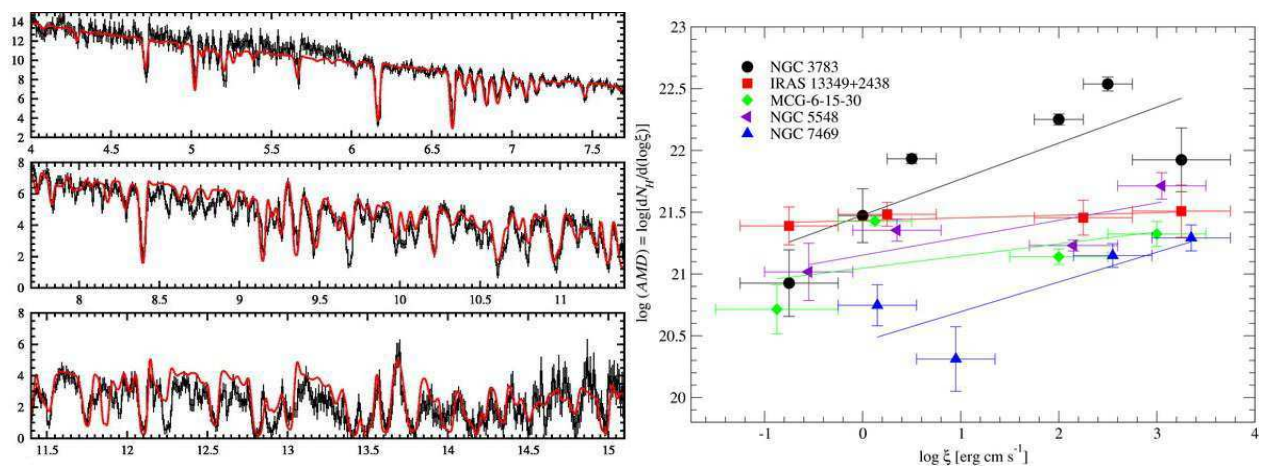

Figure 1: (a) Chandra/HETGS spectrum of NGC 3783 in [?]. (b) The derived AMDs for Seyfert AGN in $[1]$.

A large number of intensive efforts has been made to characterize the detected absorbers in UV and X-ray most relevant for exploring AGN environment in the vicinity of a central engine, while details are often rudimentary. According to UV spectroscopies primarily with HST observations of Seyfert AGNs as well as distant quasars, the presence of intrinsic absorbers in AGNs seem to be ubiquitous for more than $\sim 50 \%$ of Seyferts and quasars (e.g. $[3,4])$. Furthermore, a small fraction $(\sim 10 \%)$ of the radio-quiet quasars show substantially blueshifted UV resonance lines, referred to as broad-absorption-lines (BALs; these are mainly $\mathrm{C}$ IV/ Mg II(high/low ionization) at velocities of $v / c \sim 0.04-0.1$, where $c$ is the speed of light (e.g., [4]). Similarly in X-ray band, ionized absorbers have also been unambiguously identified with the similar fraction [11] and systematic analyses have implied that there could be an underlying physical connection between UV and X-ray outflow components [12]. These X-ray absorbers, known as warm absorbers (WAs), have a typical column density on the order of $10^{21}-10^{22} \mathrm{~cm}^{-2}$ outflowing at the line-of-sight velocity of a few $\mathrm{km} \mathrm{s}^{-1}$ to a few thousand $\mathrm{km} \mathrm{s}^{-1}$ with a vast range of ionization parameter $\xi \equiv L_{\text {ion }} /\left(n r^{2}\right) \sim 0.1-10^{4}$ erg $\mathrm{cm} \mathrm{s}^{-1}$ depending on the charge state of ions (e.g., from neutral Fe to Fe XXVI; see Fig. 1a). They are presumably originating from sub-parsec to parsec scales.

One of the powerful outflow diagnostics often utilizes the absorption measure distribution (AMD) which tells us column density distribution $N_{H}$ per decade of $\xi$ for various ions. The AMD analyses in general seem to point out to the presence of underlying continuous flow with roughly 
a constant column, to zero-th order, on which many elements of distinct charge state emerge as absorption line signatures, while there also exists an unknown variation in column distribution. The overall density profile of the absorber can thus be expressed in the power-law form as $n(r) \propto r^{-p}$ [5] where $p \sim 1$ may be the case for a number of radio-quiet Seyferts [1,9] (see Fig. 1b). If the observed wind indeed obeys $N_{H} \sim$ const profile, then this leads to a very interesting global property of the absorbers; i.e. the number density of the wind scales as $n \propto r^{-1}$ and $\xi \propto r^{-1}$ which would otherwise be $n \propto r^{-2}$ in the case of a more spherically-ejected wind such as radiation-driven winds. The wind with $n \propto r^{-1}$ profile also indicates interesting consequences in terms of AGN feedback process; i.e. outflow mass rate goes as $\dot{M}_{\text {out }} \propto r^{1 / 2}$ assuming the radial dependence of the wind velocity follows the escape velocity which is Keplerian such that $v \propto r^{-1 / 2}$. Hence, momentum rate stays constant as $\dot{P}_{\text {out }} \sim$ const, but kinetic power of the wind goes as $\dot{E}_{\text {out }} \propto r^{-1 / 2}$. This means that the mass loss rate via wind is dominated at large distances while the wind at smaller radii (i.e. closer to the central engine) makes a dominant contribution to the total energy delivered to the ambient region (if not all the way to the host galaxy). While very challenging to observationally disentangle different launching mechanisms for winds, such a global nature of the absorbers could provide a clue.
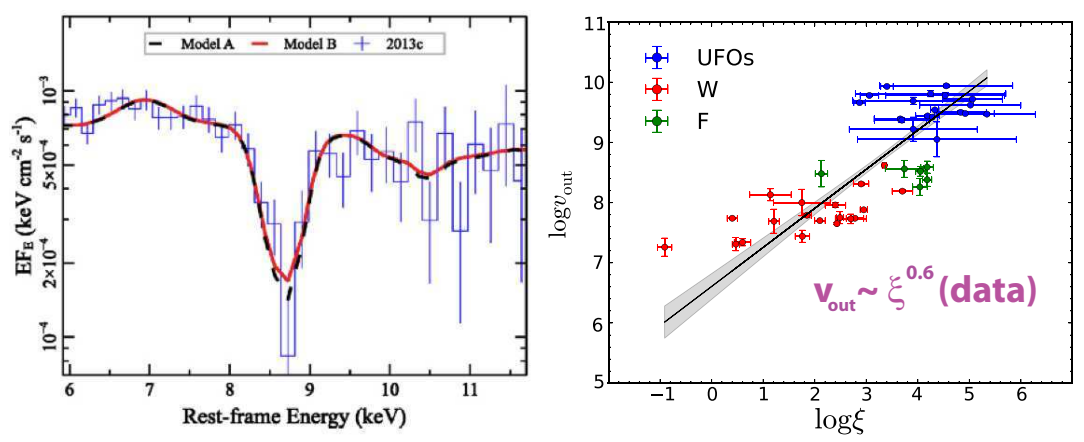

Figure 2: (a) Suzaku/XIS spectrum of PDS 456 showing the Fe K $\alpha$ UFO. (b) A likely correlation between the WAs and UFOs in [13].

Besides the long-known AGN WAs, more detailed X-ray analyses have recently revealed another distinct absorption component called the ultra-fast outflows (UFOs) from an increasing number of AGN populations (both in radio-loud/quiet ones) as well as distant lensed quasars; i.e. these outflows, typically identified almost exclusively in $\mathrm{Fe} \mathrm{K}$ band as $\mathrm{H} / \mathrm{He}$-like $\mathrm{Fe}$ absorbers, exhibit a more massive column density of $N_{H} \sim 10^{23}-10^{24} \mathrm{~cm}^{-2}$ at sub-to-near relativistic speed of $v / c \sim 0.1-0.7$ almost always characterized by high ionization parameter of $\log \xi \sim 3-5$ with a little exception so far (see Fig. 1a). A likely physical connection between the soft X-ray WAs and the Fe K UFOs has been one of the exciting subjects to further investigate and there have been some observational indications that the two components may seemingly appear to be distinct but actually belong to the same continuous AGN-driven outflows [13] (see Fig. 2b).

\section{MHD-Driven Disk-Winds in X-Ray}

Considering the observational implication of $n \propto r^{-1}$, therefore, one can construct a corresponding wind model. To this end, we have proposed a magnetically-driven wind model that is 

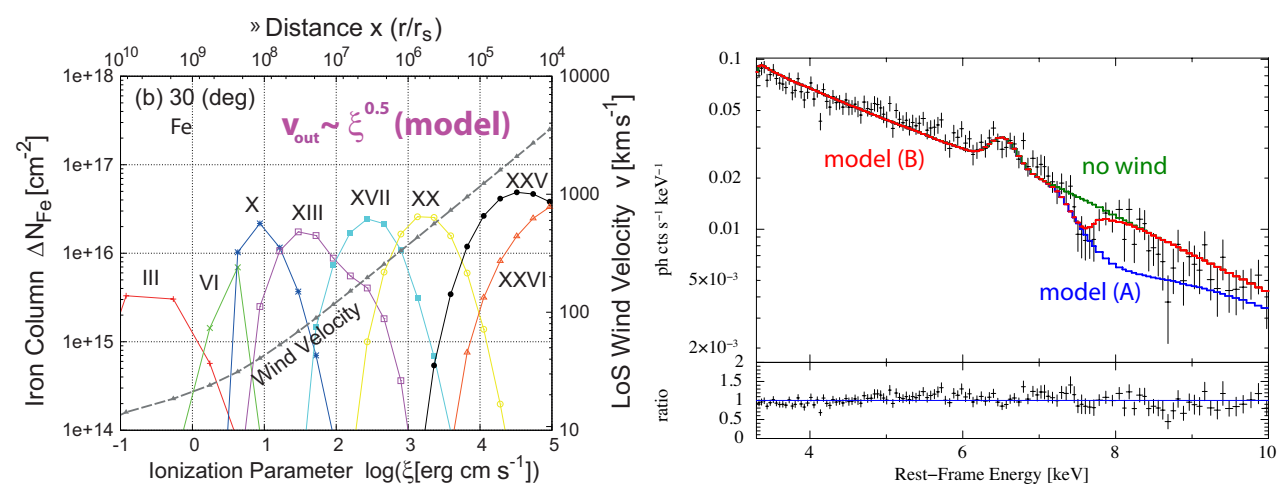

Figure 3: (a) A calculated AMD for a typical radio-quiet Seyfert with the MHD-driven disk-wind model showing a constant Fe $N_{H}$ as seen in data. (b) $60 \mathrm{ks}$ XMM-Newton/EPIC-pn spectrum of PG 1211+143 fitted with the best-fit model (B) (red) from [8].

launched and accelerated by the action of a global magnetic field in the context of magnetohydrodynamics (MHD) under Newtonian gravity without radiation pressure. This model is motivated by the following facts; (1) the derived AMD from a number of Seyfert AGN data implying $n \propto r^{-1}$ and (2) the magnetized wind is most energetically stable (with minimum magnetic energy configuration) for $n \propto r^{-1}$ [2,6]. Following the detailed formalism of self-similar MHD wind in [2] and [6], the basic equations of this problem are those of steady-state, non-relativistic, ideal MHD that include gravity and gas pressure, namely

$$
\begin{aligned}
\vec{\nabla} \cdot(\rho \mathbf{v})=\mathbf{0} & \text { (mass conservation) }, \\
\vec{\nabla} \times \mathbf{B}=\frac{\mathbf{4} \pi}{\mathbf{c}} \mathbf{J} & \text { (Ampere's law) }, \\
\mathbf{E}+\frac{\mathbf{v}}{\mathbf{c}} \times \mathbf{B}=\mathbf{0} & \text { (ideal MHD) }, \\
\vec{\nabla} \times \mathbf{E}=\mathbf{0} & \text { (Faraday's law) } \\
\rho(\mathbf{v} \cdot \nabla) \mathbf{v}=-\vec{\nabla} \mathbf{p}-\rho \vec{\nabla} \mathbf{V}_{\mathbf{g}}+\frac{\mathbf{1}}{\mathbf{c}}(\mathbf{J} \times \mathbf{B}) & \text { (momentum conservation) } \\
\vec{\nabla} \cdot \mathbf{B}=\mathbf{0} &
\end{aligned}
$$

where the plasma wind velocity $\mathbf{v}$ is assumed to be frozen into the global magnetic field $\mathbf{B} ; \mathbf{E}$ is the electric field; $\mathbf{J}$ is the electric current density; and $c$ is the speed of light. The radial extent is assumed by self-similar prescription such that magnetic stream function is expressed in the powerlaw form; $\Psi(r, \theta) \propto r^{q} f(\theta)$ where $f(\theta)$ is the polar angular dependence to be numerically solved by the Grad-Shafranov equation. For $n \propto r^{-1}$ profile, we choose $q=1$. The wind kinematics is therefore completely described in the MHD framework. Radiative transfer through plasma, on the other hand, is calculated with xstar photoionization code in which heating (photoelectric and collisional) and radiative cooling balance is solved under ionization equilibrium and we have exploited a computational scheme appropriate for our MHD accretion-disk wind model $[6,7]$.

The model has been applied to a number of cases and successfully described the major characteristics of the observed absorption features. First, it was applied to explain the observed AMD relevant to typical Seyferts [6]. Assuming a typical ionizing AGN spectrum of power-law-dominant 
SED with $\Gamma=2$, the calculated AMD for major ions (Fig. 3a) is indeed found to be almost constant over $4-5$ decades in $\xi$ with highly-ionized elements at higher velocity while weakly-ionized ones at lower velocity in consistence with data.

This model can also be applied to further explain the UFOs. As a case study, we investigated a soft-X-ray-luminous AGN, PG $1211+143$, by properly adjusting its SED; i.e. a multi-color disk radiation plus a power-law component [8] such that X-rayto-UV flux ratio (at $2 \mathrm{keV}$ and $2500 \AA$ ) is $\alpha_{\mathrm{ox}}=-1.5$ (see NED). As in [6], photoabsorption cross section $\sigma_{\mathrm{pi}}$ of each line transition is calculated from ionic column $N_{\text {ion }}$ through xstar code coupled to an obtained matter field. The line spectra are calculated using the Voigt function. The model provides an excellent fit $\left(\chi^{2} / \mathrm{dof}=198.54 / 128\right)$

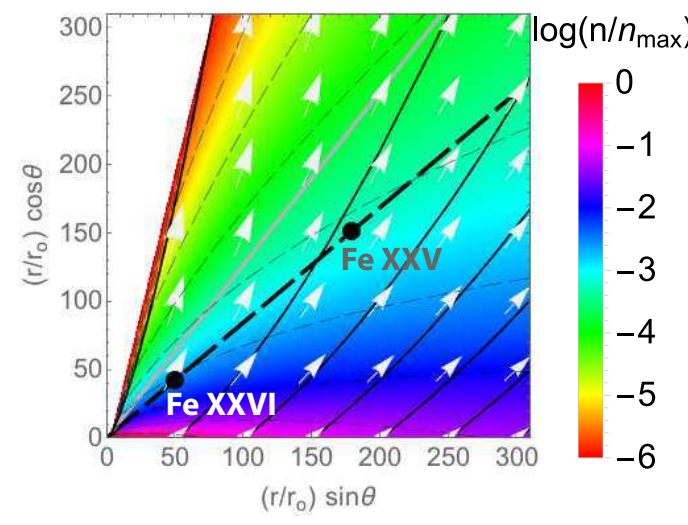

Figure 4: A poloidal representation of the calculated MHD disk-wind superimposed to the derived position of the observed Fe XXV and Fe XXVI in [8]. to a 60-ks XMM-Newton/EPIC-pn spectrum of PG 1211+1433 indicating a number of observational constraints on the $\mathrm{Fe} \mathrm{K}$ absorber in the context of the MHD-driven wind scenario for this source; namely, the detected absorber is identified as Fe XXV of the LoS column density of $N_{H}(\mathrm{Fexxv}) \sim 1.2 \times 10^{23} \mathrm{~cm}^{-2}$ with the inclination angle of $\theta_{\mathrm{obs}} \sim 50^{\circ}$, the absorber's LoS characteristic velocity is $v_{\mathrm{Fexxv}} 0.115 \mathrm{c}$ at its characteristic distance of $r_{\mathrm{Fexxv}} \sim 200 R_{S}$ from the central engine, and the characteristic ionization parameter is derived to be $\log \xi \sim 5.31$ (see model (B) in Fig. 3b). Due to the line width in data, the model requires the inner edge of the disk of the MCD temperature of $k T \sim 38 \mathrm{eV}$ to be truncated at $r_{\text {trun }} \sim 30 R_{S}$ (where $R_{S}=2 G M / c^{2}$ is the Schwarzschild radius) in such a way that the contribution from Fe XXVI is appropriately minimized [8]. The derived parameters of the Fe XXV UFO in PG 1211+143 further indicates that the mass-outflow rate is $\dot{M}_{\text {out }} \sim 2.56 \mathrm{M}_{\odot} /$ yr which may be well sufficient to make an impact on the AGN feedback at least in part. This is also consistent with the earlier estimates based on a phenomenological xstar modeling in the literature. In this view the spatial location of the absorbers is depicted in Figure 4 where the calculated wind is superimposed. Its global property is also discussed further in detail in [10].

\section{Summary}

AGN-driven outflows have been ubiquitously found across diverse AGN populations in the past decades. Particularly in X-ray regime there is an increasing number of circumstantial evidences that the UV and X-ray winds are most likely connected in various ways. Furthermore, within X-ray absorbers themselves, there seems to exist a different types of absorbers in terms of their column densities, velocities and ionization state. The underlying physics of these "seemingly distinct diversity" in the observed winds remains unclear today. Among other wind models available in the literature, we propose a magnetically-driven disk-wind model. One of the fundamental 
generic feature of the model is that the high-velocity layer (i.e. UFOs) is always present in the wind at its innermost part in highly-ionized state regardless of the exact AGN SED (including the X-ray luminosity). For a given ionizing flux (i.e. hard X-ray photons relative to UV-to-soft$\mathrm{X}$-ray ones), this high-velocity portion of the wind at smaller distances can be completely ionized thus spectroscopically undetectable while in fact present. The slower portion of the wind at intermediate-to-large distances (i.e. WAs) is always visible with a moderate value of the ionization parameter. In this scenario, therefore, $\alpha_{\mathrm{Ox}}$ may play a crucial role in allowing the absorbers to be either detectable or not.

\section{References}

[1] Behar, E. Density Profiles in Seyfert Outflows, ApJ 703 (2009) 1346

[2] Contopoulos, J., Lovelace, R. V. E. Magnetically driven jets and winds: Exact solutions, ApJ 429 (1994) 139

[3] Crenshaw, D. M., Kraemer, S. B., Boggess, A., et al., Intrinsic Absorption Lines in Seyfert 1 Galaxies. I. Ultraviolet Spectra from the Hubble Space Telescope, ApJ 516 (1999) 750

[4] Crenshaw, D. M., Kraemer, S. B., George, I. M, Mass Loss from the Nuclei of Active Galaxies, ARA\&A 41 (2003) 117

[5] Detmers, R. G., Kaastra, J. S., Steenbrugge, K. C., et al. Multiwavelength campaign on Mrk 509. III. The 600 ks RGS spectrum: unravelling the inner region of an AGN, A\&A 534 (2011) 38

[6] Fukumura, K., Kazanas, D., Contopoulos, I., et al. Magnetohydrodynamic Accretion Disk Winds as X-ray Absorbers in Active Galactic Nuclei, ApJ 715 (2010a) 636

[7] Fukumura, K., Kazanas, D., Contopoulos, I., et al. Modeling High-velocity QSO Absorbers with Photoionized Magnetohydrodynamic Disk Winds, ApJL 723 (2010b) 228

[8] Fukumura, K., Tombesi, F., Kazanas, D., et al. Magnetically Driven Accretion Disk Winds and Ultra-fast Outflows in PG 1211+143, ApJ 805 (2015) 17

[9] Holczer, T., \& Behar, E. X-Ray Absorption Analysis of NGC 3516: Appearance of Fast Components with Increased Source Flux, ApJ 747 (2012) 71

[10] Kazanas, D., Fukumura, K., Behar, E., et al., Toward a Unifed AGN Structure, AstRv 7 (2012) 92

[11] Reynolds, C. S., An X-ray spectral study of 24 type 1 active galactic nuclei, MNRAS 286 (1997) 513

[12] Steenbrugge, K. C.; Kaastra, J. S.; Crenshaw, D. M., et al., Simultaneous X-ray and UV spectroscopy of the Seyfert galaxy NGC 5548. II. Physical conditions in the X-ray absorber, A\&A 434 (2005) 569

[13] Tombesi, F., Cappi, M., Reeves, J. N., et al., Unification of X-ray winds in Seyfert galaxies: from ultra-fast outflows to warm absorbers, MNRAS 430 (2013) 1102 